\title{
Systematic Evaluation of Skeletal Mechanical Function
}

\author{
Lauren Smith, ${ }^{1}$ Erin M.R. Bigelow, ${ }^{1}$ and Karl J. Jepsen ${ }^{1}$ \\ ${ }^{1}$ The University of Michigan, Ann Arbor, Michigan
}

\begin{abstract}
Many genetic and environmental perturbations lead to measurable changes in bone morphology, matrix composition, and matrix organization. Here, straightforward biomechanical methods are described that can be used to determine whether a genetic or environmental perturbation affects bone strength. A systematic method is described for evaluating how bone strength is altered in the context of morphology and tissue-level mechanical properties, which are determined in large part from matrix composition, matrix organization, and porosity. The methods described include computed tomography, whole-bone mechanical tests (bending and compression), tissue-level mechanical tests, and determination of ash content, water content, and bone density. This strategy is intended as a first step toward screening mice for phenotypic effects on bone and establishing the associated biomechanical mechanism by which function has been altered, and can be conducted without a background in engineering. The outcome of these analyses generally provides insight into the next set of experiments required to further connect cellular perturbation with functional change. Curr. Protoc. Mouse Biol. 3:39-67 (c) 2013 by John Wiley \& Sons, Inc.
\end{abstract}

Keywords: bone $\bullet$ biomechanics $\bullet$ strength $\bullet$ nanocomputed tomography $\bullet$ cortical bone $\bullet$ trabecular bone $\bullet$ adaptation

\section{INTRODUCTION}

The skeletal system is affected by many genetic and environmental perturbations, often leading to measurable changes in bone morphology, matrix composition, and matrix organization. Oftentimes, investigators stop here, drawing conclusions about the role of a gene, for example, based on these structural and compositional alterations. However, these associations reveal only part of the story. First, it is important to recognize that many perturbations affect body mass, and that adjusting traits for body mass is essential, because larger mice generally have larger bones. The challenge is to determine whether changes in a trait are greater (or less) than expected for the effect on body mass. Second, to determine whether a genetic or environmental perturbation also affects bone strength requires additional knowledge of the complex adaptive nature of the skeletal system. Because the skeletal system is highly adaptive, perturbations that affect one trait are often accompanied by compensatory changes in other traits. Such adaptation must be considered when attempting to draw meaningful conclusions about the role of a gene or other perturbation. The challenge here is to figure out which alterations can be attributed directly to the perturbation and which result from adaptive changes associated with establishing and maintaining mechanical function.

The following protocols describe straightforward biomechanical methods that can be used to determine whether a genetic or environmental perturbation affects bone strength. They provide a systematic method for evaluating how bone strength is altered in the context of morphology (bone size and shape) and tissue-level as well as whole-bone mechanical properties, which are determined in large part from matrix composition, matrix organization, and porosity. This strategy is a first step for screening mice for phenotypic

Systematic Evaluation of Skeletal

Mechanical Function 
effects and establishing the associated biomechanical mechanism by which function is altered. Basic Protocols 1 and 2 describe harvesting and embedding of bone. Basic Protocol 3 describes imagined by nanocomputed tomography (nanoCT). Basic Protocol 4 and Alternate Protocols 1 and 2 present methods for assessing whole-bone mechanical properties using bending and compression tests. Basic Protocol 5 describes tissue-level mechanical tests, and Basic Protocol 6 describes measurement of ash content, water content, and bone density. Basic Protocol 7 describes a systematic approach for comparing functional and morphological properties of bones from control and experimental groups.

These protocols were written to enable researchers without a background in engineering to conduct assays, as well as to provide a better understanding of outcomes from similar assays conducted by colleagues knowledgeable in engineering. Additional testing is often required (e.g., FTIR, Raman, Nano-indentation), and there are many resources that can help with these methods.

CAUTION: Gloves should always be worn when handling laboratory animals. Gloves and goggles should be worn when handling chemicals.

BASIC PROTOCOL 1

Systematic Evaluation of Skeletal Mechanical Function

\section{HARVESTING BONE}

Harvesting bones is relatively simple, but must be done carefully to avoid breaking them. A basic understanding of anatomy is required to disarticulate and extract a bone without damaging the epiphyses, which can easily be sheared off in younger mice.

NOTE: All protocols involving live animals must be reviewed and approved by an Institutional Animal Care and Use Committee (IACUC) and must conform to government regulations for the care and use of laboratory animals.

\section{Materials}

\author{
Mouse \\ $1 \times$ phosphate-buffered saline $(\mathrm{PBS})$ \\ $10 \%$ neutral buffered formalin (NBF) \\ 2-ml microcentrifuge tubes \\ Permanent marker \\ Freezer storage boxes, cardboard or plastic \\ Gauze \\ Surgical tools \\ Tissue cassettes (Histosette II, Fisher Scientific) \\ No. 2 pencil \\ 1-liter wide-mouth HDPE bottles (Qorpak) \\ Absorbent pads
}

Additional reagents and equipment for euthanasia (Donovan and Brown, 2006)

\section{For imaging or mechanical testing}

1a. Label 2-ml microcentrifuge tubes using a permanent marker. Include the animal identification number and bone on the top and side of each tube (Fig. 1).

2a. Label freezer storage boxes using a permanent marker. Include the animal identification numbers and type of bone.

It is useful to separate bones by type, i.e., with femurs in one box, spines in another, etc. In this case, animal identification numbers can be grouped (e.g., B6 1-10).

3a. Cut gauze into long pieces that can wrap around an entire bone and soak them in PBS.

4a. Record body mass of the mouse before starting dissection. 


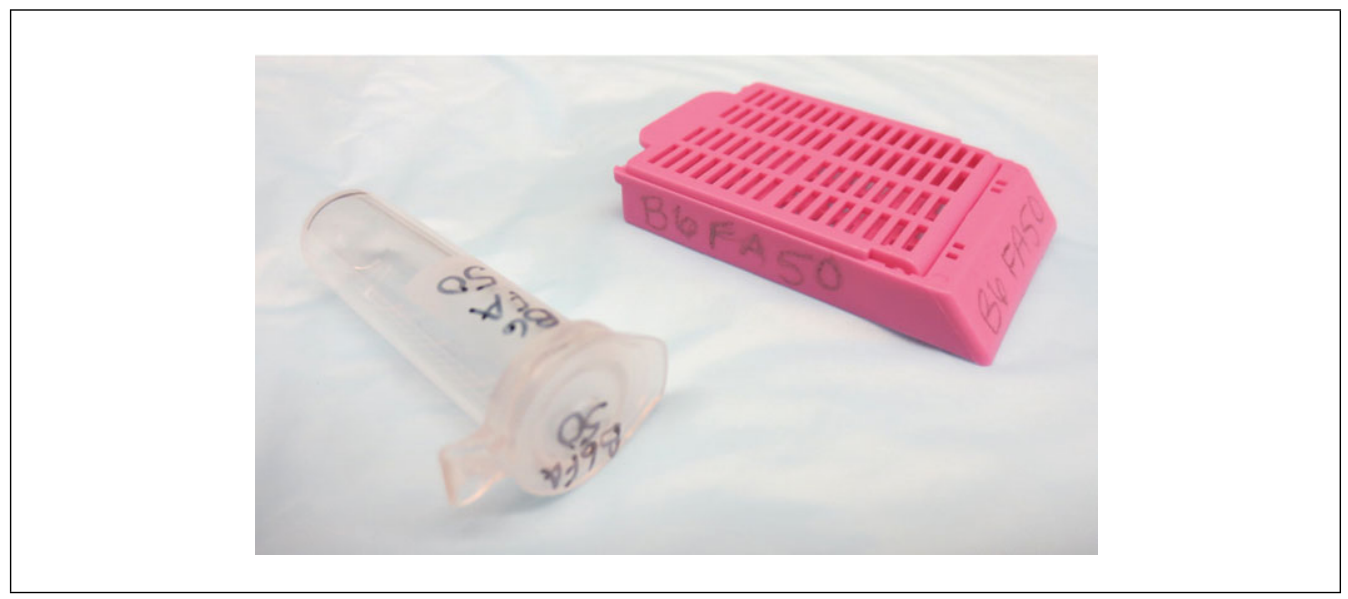

Figure 1 Proper marking of microcentrifuge tube with permanent marker and tissue cassette with no. 2 pencil.

5a. Sacrifice mouse according to experimental need and institutional guidelines.

6a. Harvest bones, working from distal to proximal (e.g., remove the tibia before the femur). Be careful to avoid scratching the bones.

The tools used to harvest bones are a matter of personal preference. Some prefer to use a \#15 blade, others a \#11 blade to more easily get underneath tendons, and some prefer to use dissection scissors. A small pair of tweezers can be helpful to hold tissue while removing bones.

7a. Remove as much soft tissue from the bones as possible using gauze. If a scalpel must be used, be careful not to cut or scratch the bone surface. Be careful not to disturb the periosteum.

8a. Wrap each bone completely in PBS-soaked gauze, then place in the appropriately labeled 2-ml tube.

Alternatively, place the unwrapped bone in the tube and then fill the tube with PBS, making sure the entire bone is covered, but leaving enough room for the PBS to expand with freezing.

9a. Close the tube so it is sealed and place in the appropriately labeled freezer storage box.

10a. When done, place all freezer storage boxes in a $-20^{\circ}$ or $-40^{\circ} \mathrm{C}$ freezer.

When many dissections are being done, bones may be collected at room temperature for up to 4 to $5 \mathrm{hr}$ before they should be moved to the freezer. Once frozen, bones may be stored up to 6 months. With longer storage, the long bones may become freezer burned.

\section{For histology}

1b. Label tissue cassettes using a No. 2 pencil. Include the animal identification number and the bone in two places on each cassette in case one is rubbed off or smudged (Fig. 1).

CAUTION: Never use pen or permanent marker, as the chemicals used for plastic or paraffin embedding will remove the label and you will have no way to identify your specimens. Entire research studies have been lost because of labeling with pen.

2b. For every 20 cassettes, fill a 1-liter bottle with $10 \%$ NBF (or other fixation agent that works with the desired histological processing method). Place bottles on an absorbent pad, fill with NBF, and affix a permanent label to the outside of each bottle, indicating the fixative (e.g., 10\% NBF), animal identification numbers, and bone type. Keep bottles tightly closed until ready to use.

Systematic Evaluation of Skeletal

Mechanical

Function

\section{1}

Volume 3 
CAUTION: Wear gloves and safety goggles and work under a chemical fume hood.

It is useful to separate bones by type, i.e., with femurs in one bottle, spines in another, etc. In this case, animal identification numbers can be grouped (e.g., B6 1-10).

3b. Record body mass of the mouse before starting dissection.

4b. Sacrifice mouse according to experimental need and institutional guidelines.

5b. Harvest bones and remove soft tissue (see steps 6a-7a).

6b. Place bones in the appropriately labeled histology cassettes.

7b. Place cassettes in the appropriately labeled fixative bottles.

$8 \mathrm{~b}$. When done, store all bottles away from direct sunlight and out of heavy traffic. Ensure that shelves are sturdy enough to support the weight, especially if there are many bottles.

Bones should be stored in fixative for no more than several days. For longer periods, they be washed and stored in another chemical (e.g., 70\% ethanol). Excessive storage in fixative may cause bones to degrade.

BASIC PROTOCOL 2

Systematic Evaluation of Skeletal Mechanical Function

\section{EMBEDDING BONE IN PLASTIC}

Bone samples can be embedded in plastic or paraffin to evaluate changes in matrix organization arising from a genetic or environmental perturbation. In addition, histomorphometry can be performed to provide information on changes in bone formation rates that may account for structural alterations associated with a genetic or environmental perturbation. To conduct histomorphometric analyses, bone samples must be embedded in plastic without prior decalcification. The following is a two-step embedding process, which helps to center the bone in the plastic cylinder.

The fluorescent labels used to mark new bone deposition are incorporated into the bone mineral. These labels are removed upon decalcification. Many fluorescent labels are available, and the choice of label can be based on the filters available for the epifluorescence microscope. We inject each mouse with $30 \mathrm{mg} / \mathrm{kg}$ body weight of Calcein at 7 days prior to sacrifice plus $30 \mathrm{mg} / \mathrm{kg}$ Alizarin-red at 2 days prior to sacrifice (both at $10 \mathrm{mg} / \mathrm{ml}$ ). This allows 5 days between labels, and is sufficient for mice less than 12 weeks of age. After 12 weeks of age, bone formation has slowed quite a bit, so the time between injections can be increased to make sure there is sufficient space between the labels to generate accurate measures of mineral apposition rate.

\section{Materials}

Bone samples in tissue cassettes (see Basic Protocol 1)

$10 \%$ neutral buffered formalin (NBF)

$1 \times$ phosphate-buffered saline $(\mathrm{PBS})$ or distilled water

Villanueva Osteochrome Bone Stain (Polysciences, optional)

Methanol (optional)

Ethylene glycol monoethyl ether (EGME)

2-Propanol

Methyl salicylate, $99 \%$

Methyl methacrylate (MMA) solutions I-IV (see Support Protocol)

Cyanoacrylate

Diamond slurries (1-2, 6, and $10 \mu \mathrm{m})$

1-liter wide-mouth HDPE bottles (Qorpak)

Agitator

5-ml plastic vials with caps 
Vacuum oven (Isotemp or Eurotherm 91e Blue, Fisher Scientific)

Small drill

Small wooden dowel rod

Cutting device: commercial metallurgical cutting system with diamond wafering blade (e.g., Isomet Low-Speed Saw, Buehler)

$1 \times 3$-in. plastic slides

Sandpaper ranging from 600 to 1000 grit

Light microscope (Axioplan2, Carl Zeiss IMT) or epifluorescence microscope

CAUTION: DO NOT open bottles of MMA II, III, or IV until they have been warmed completely to room temperature. Water vapor may condense on the surface of cold MMA when exposed to normal, humidified air, which will introduce water into the solution and compromise the embedding procedure.

\section{Fix samples}

1. Submerge specimens in tissue cassettes in $10 \%$ NBF in a 1-liter wide-mouth bottle for $24-48 \mathrm{hr}$ at room temperature.

Samples may be left in NBF at room temperature for up to several months, but should be moved to $70 \%$ ethanol or $1 \times P B S$ for longer storage. If specimens are stored in ethanol, wash twice for $12 \mathrm{hr}$ each with PBS or water at $4^{\circ} \mathrm{C}$ before proceeding to the next step.

\section{Pre-stain with Osteochrome (optional)}

2. Soak in Villanueva Osteochrome Bone Stain for $48 \mathrm{hr}$.

Osteochrome is a bulk stain used to visualize osteoids, cells, and microstructure.

3. Wash twice in $100 \%$ methanol for at least $2 \mathrm{hr}$ each to remove any excess stain.

\section{Dehydrate}

4. Pre-rinse with EGME. If coming directly from NBF, rinse for at least $12 \mathrm{hr}$. If coming from ethanol or PBS, this time can be shortened.

It is important to avoid introducing water into the system from this step forward.

This procedure can be automated using a programmable plastics processing system. The total dehydration time is $48 \mathrm{hr}$.

5. Dehydrate samples in four changes of EGME for $4 \mathrm{hr}$ each, using separate bottles labeled 1 thru 4.

To save chemicals, after dehydrating $\sim 20$ bones, rotate bottle 2 to bottle 1, bottle 3 to bottle 2, and bottle 4 to bottle 2. Discard bottle 1 and use a new bottle with fresh EGME as the new bottle 4. Continue this rotation process. The logic here is that the first change of EGME will be the most contaminated with ethanol.

6. Rinse in three changes of 2-propanol for $4 \mathrm{hr}$ each.

7. Clear using two changes of methyl salicylate for $4 \mathrm{hr}$ each.

To save chemicals, the above rotation method can be used.

\section{Embed specimens}

8. Submerge in MMA I for at least $48 \mathrm{hr}$. If desired, place on an agitator at medium speed to increase infiltration.

9. Submerge in room temperature MMA II and then agitate for at least $48 \mathrm{hr}$ at $4{ }^{\circ} \mathrm{C}$.

10. Submerge in room temperature MMA III and then agitate for at least $48 \mathrm{hr}$ at $4{ }^{\circ} \mathrm{C}$.

\section{Create base for embedding}

11. For each sample, fill a 5-ml vial just slightly less than half way with room temperature MMA IV. Spread the plastic along one side of the vial vertically, creating a half cylinder. 

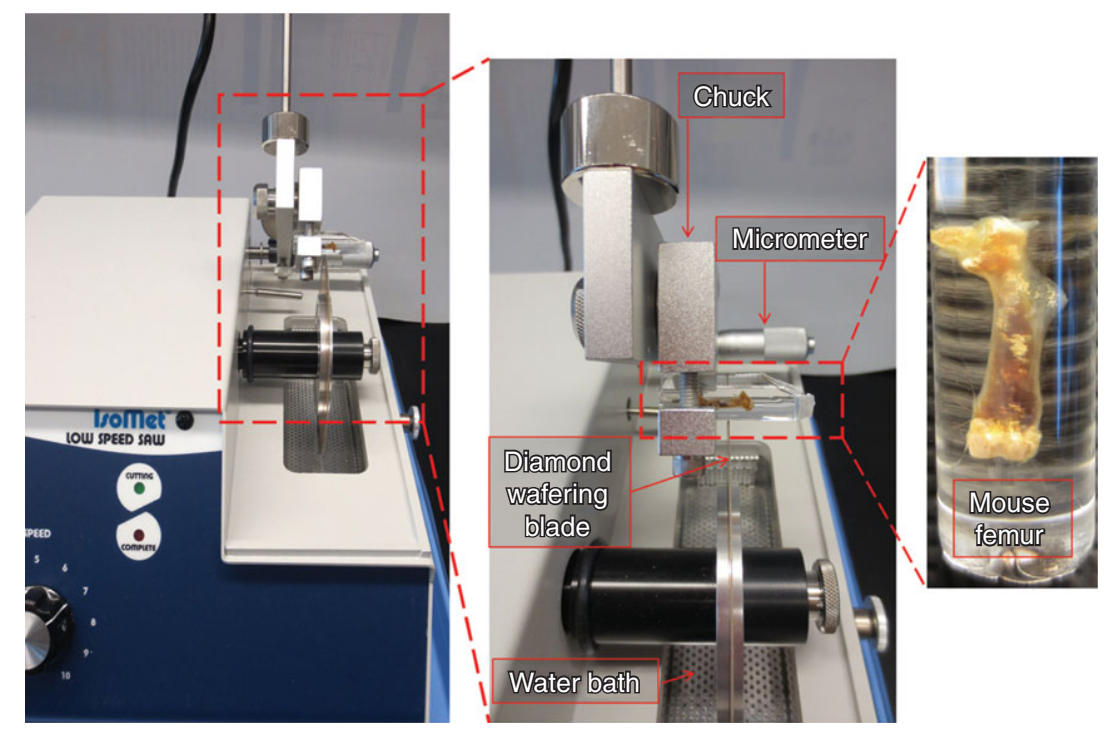

Figure 2 Sectioning of mouse femora. (A) Wide view of IsoMet diamond wafering saw. (B) Enlarged view of chuck, specimen, and blade interface. (C) Single femur embedded in polymerized MMA.

12. Tighten caps on the vials and place vials on their sides in a $37^{\circ} \mathrm{C}$ oven. Leave space between the vials so air can circulate during curing, and secure them so they cannot roll. Leave in the oven until the half cylinder of plastic has hardened (1-2 days).

\section{Place samples in base}

13. Add samples in MMA IV to the hardened bases, centering and orienting the bones with the vertical axis of the vials. Ideally, place the anterior surface of the bone, being the flattest side, on the hardened plastic half.

14. To remove bubbles, place vials on their sides with the caps off in a vacuum for $10 \mathrm{~min}$.

Bubbles should rise out of the sample, resulting in a translucent plastic.

15. Secure caps on the vials and store at room temperature for 48-72 $\mathrm{hr}$ to continue the polymerization process while preventing further bubble formation.

16. Transfer to a $37^{\circ} \mathrm{C}$ oven for 3-4 days for final hardening.

\section{Section and mount samples}

17. Carefully remove the sample from the plastic vial by drilling a small hole in the bottom of the vial and gently pushing the sample out with a dowel.

18. Place the bone in the cutting device, orient the sample so the blade sections it transversely, and then secure it with a chuck (usually supplied by the manufacturer; Fig. 2).

19. Cut 200- $\mu \mathrm{m}$ sections until there are a sufficient number of sections to represent the region of interest.

For mouse femurs, cut the bone just distal to the third trochanter. It will take four to five sections to sample the mid-shaft.

Systematic Evaluation of Skeletal

Mechanical Function

Sections that are $200 \mu \mathrm{m}$ thick are easily handled. If the sections are too thin, routine handling can flex the sections, which often results in cracking. 
20. Polish sections lightly on one side using 600- to 800-grit sandpaper.

21. Glue the polished side to a $1 \times 3$-in. plastic slide using cyanoacrylate.

22. Grind and polish the slide using a series of sandpapers (600 to 1000 grit) followed by a series of diamond slurries (10,6, and 1-2 $\mu \mathrm{m})$.

The final sample thickness should be $\sim 30$ to $50 \mu \mathrm{m}$.

23. View sections using a light or epifluorescence microscope.

\section{PREPARATION OF METHYL METHACRYLATE SOLUTIONS}

CAUTION: Work in a well-ventilated chemical fume hood and wear goggles, gloves, a mask, and a lab coat. Use separate funnels and graduated cylinders designated specifically for MMA or $n$-butyl phthalate. DO NOT use a metal scoop when weighing benzoyl peroxide, as this may cause a spark. Use only a wooden spatula. Use caution when transporting benzoyl peroxide and do not breathe the fumes.

\section{Materials}

Methyl methacrylate (MMA)

$n$-Butyl phthalate ( $n$-BP)

Dry benzoyl peroxide

1-liter wide-mouth HDPE bottles (Qorpak)

Orbital shaker

1. Add $765 \mathrm{ml}$ MMA to each of four 1-liter bottles labeled (with permanent marker) as MMA I to IV.

2. Add $140 \mathrm{ml} n$-BP to each bottle.

3. Add the following amounts of dry benzoyl peroxide to each bottle:

$\begin{array}{ll}\text { MMA I } & 0 \mathrm{~g} \\ \text { MMA II } & 9.0 \mathrm{~g} \\ \text { MMA III } & 16.75 \mathrm{~g} \\ \text { MMA IV } & 17.75 \mathrm{~g} .\end{array}$

If benzoyl peroxide is hydrated rather than dry, add 25\% more by weight.

4. Mix on an orbital shaker for $1 \mathrm{hr}$.

MMA I-III are complete after mixing. MMA I can be stored at room temperature in a cabinet for flammables. MMA II and III can be stored at $-20^{\circ} \mathrm{C}$.

5. Thicken MMA IV in a $37^{\circ} \mathrm{C}$ water bath. Monitor progress consistently (no less than every $30 \mathrm{~min}$ ), shaking well each time. When the bottle begins to contract and the solution turns slightly yellow, monitor more frequently, as the process is beginning to accelerate. Continue until the solution has a consistency similar to honey.

This process usually takes a few days, but each solution behaves a little differently.

CAUTION: Thickening should be done carefully. If the reaction heats up too quickly, it may explode. If at any point the reaction is proceeding too quickly, cool the MMA bottle at $-20^{\circ} \mathrm{C}$ to pause the polymerization process.

MMA IV can be stored at $-20^{\circ} \mathrm{C}$. Once frozen, do not open any MMA bottle until thawed and warmed to room temperature. It is important to avoid introducing water into the solution, as it can interfere with the embedding process.

Systematic Evaluation of Skeletal

Mechanical

Function

45

Volume 3 
BASIC

PROTOCOL 3
Systematic Evaluation of Skeletal Mechanical Function
To determine whether a genetic or environmental perturbation affects bone morphology, bone samples can be imaged using micro-computed tomography (microCT). There are many systems on the market, and all generate three-dimensional images from which cross-sectional morphology of cortical bone and trabecular architecture can be measured. The protocol below describes a similar procedure, nanoCT, that can be used to acquire images with resolutions approaching $0.5 \mu \mathrm{m}$. Such high resolution may be useful when assessing morphological properties in young mice, which have small structures with low mineralization levels. This combination of traits makes it difficult to acquire high-fidelity images using conventional microCT systems.

The general concepts presented below can be adapted to other CT systems. However, when using closed-tube systems, it is not optimal to push the system to its limits, as this decreases the overall lifetime of the system. Open-tube systems allow greater parameter flexibility and range, because maintenance of the system is easy and relatively inexpensive.

\section{Materials}

Water or $1 \times$ phosphate-buffered saline (PBS)

Frozen bone samples (see Basic Protocol 1)

Calibration standard

Nanotom S computed tomography system (GE Sensing \& Inspection Technologies GmbH; Fig. 3)

Cylindrical specimen tube (made out of acrylic, polycarbonate, or other similar material)

Custom fixtures to hold specimen holder and acrylic equilibration bath (if needed, will depend on your design)

Bone holder (made out of acrylic, polycarbonate, or other similar material)

$1.69 \mathrm{mg} / \mathrm{cc}$ cortical bone equivalent rod, hydroxyapatite standard (Gammex)

Acrylic equilibration bath

Sonicator

Latex rubber bands

Acrylic pieces

Computers for acquisition and reconstruction (128 GB RAM; 8 CPUs; Intel Xeon 3.2 GHz; NVIDIA Graphics cards; 2 TB hard drive; Linux virtual machine)

Reconstruction software

VGStudio MAX 2.1 Software (Volume Graphics GmbH)

MicroView Advanced Bone Analysis (v. 2.2; GE Healthcare) software

Filter material $(0.25,0.3$, or $0.762 \mathrm{~mm}$ aluminum $)$

\section{Prepare fixtures for imaging system}

1. Design a cylindrical tube that can be placed on the sample stage and hold the specimen holder with bones in individual places during the scan (Fig. 4A).

The tube must be able to be securely fastened to the stage without movement and must be able to hold water or PBS without leaking (Fig. 4B,C). Acrylic is a great option for material, because it has similar attenuation characteristics as water. If using acrylic, be aware that alcohols can cause the material to crack and eventually shatter. Polycarbonate is an alternative option.

2. Design any necessary fixtures needed to hold the sample holder to ensure alignment relative to the beam and detector.

The Nanotom $S$ has a laser for centering and alignment of samples. Furthermore, if the sample is centered in the stage, it should be centered in the field of view. 


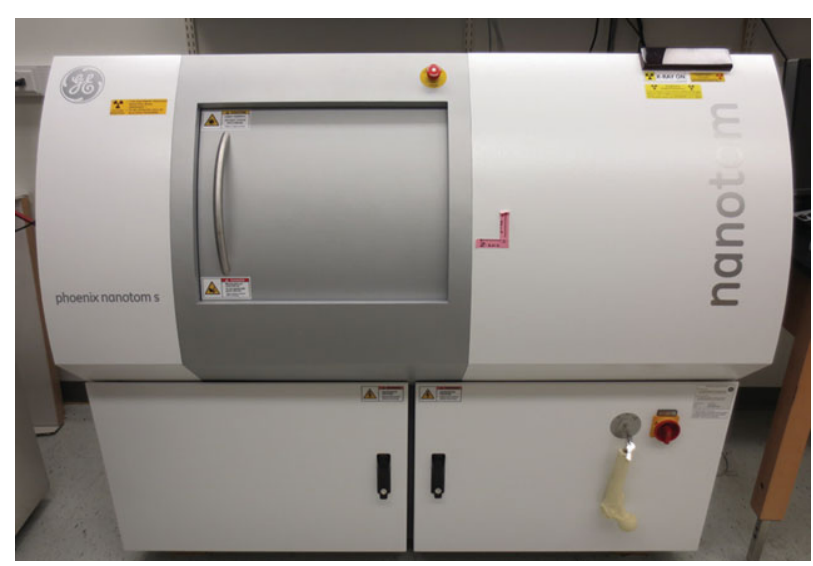

Figure 3 External view of Nanotom S nanoCT (approximately $64 \times 56 \times 29$ inches and 2750 pounds).

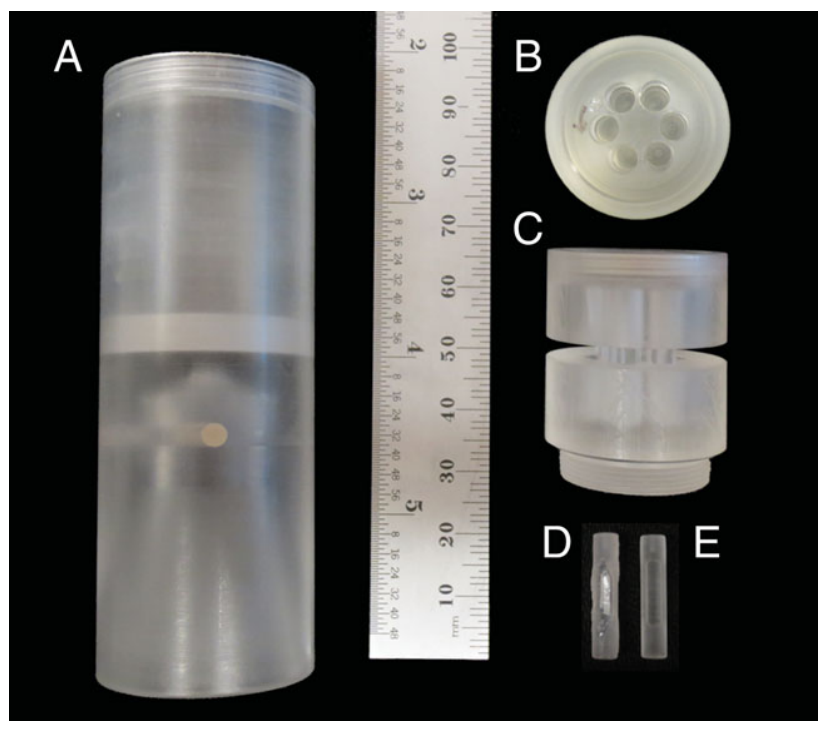

Figure 4 Five-bone scanning unit. (A) Acrylic cylindrical tube with a threaded insert. (B) Top view of six-well acrylic specimen holder. (C) Side view of same six-well specimen holder showing threads (bottom) that screw into the insert of the tube to maintain stability. This view also shows the center in-cut where a latex rubber band can be placed to further secure the bones. (D) Filled calibration insert, with hydroxyapatite standard embedded in center. (E) Empty calibration insert with a small air hole on top and solid acrylic bottom that represents water.

3. Design a calibration standard that can be incorporated in the sample holder.

The calibration standard should consist of a region of air, a region of water, and a beam of a $1.69 \mathrm{mg} / \mathrm{cc}$ hydroxyapatite (HA) phantom (Fig. 4D). This standard is placed in one well of the specimen holder. A quick-setting plastic can be used to seal the HA beam to the machined calibration standard (Fig. 4E).

4. Incorporate an equilibration bath into the system to improve signal quality.

This is an acrylic block with a central hole (Fig. 5). The cylinder holding the samples slides into this central hole. The equilibration bath creates a uniform thickness of material (acrylic, water) to improve uniformity of the X-ray beam.

Systematic Evaluation of Skeletal

Mechanical

Function 


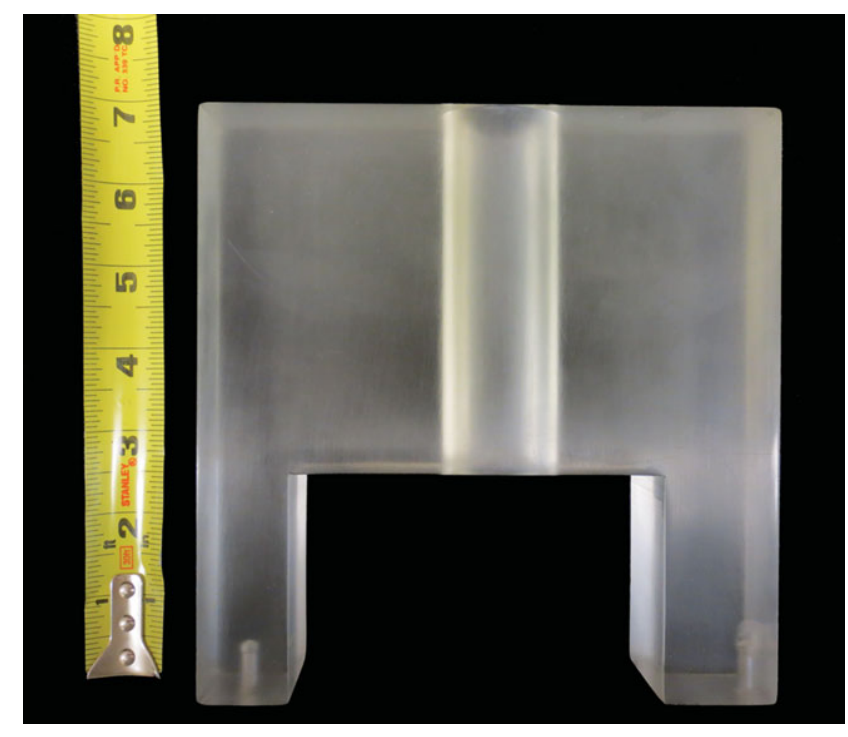

Figure 5 Acrylic equilibration block with drilled-out center for placement of the loaded cylindrical tube. Metal stick inserts on the bottom to stabilize holder on the stage of the nanoCT.

5. Eliminate all metal. Sonicate all pieces that will be visible in the scan. Avoid the use of metal utensils when placing the sample in the holder or manipulating the holder.

A small piece of metal can essentially compress all the other signals in the sample into a much smaller range of gray values, resulting in decreased bone density detectability.

\section{Load specimens}

6. Fill the sample holder and tube with water, PBS, or other solution.

This is necessary to avoid bubbles that affect scan quality. Large bubbles scatter the $X$-ray beam and cause hardening of the X-rays, which can lead to non-uniformity in the reconstructed image field.

7. Insert individual bone samples into the cylinder, securing each sample in a well of the cylinder using a latex rubber band placed tightly in the notch located in the center of the specimen holder.

Acrylic pieces of varying size and shape are helpful when stabilizing specimens of varying size.

\section{Adjust system settings}

8. Place the cylinder and holder into the instrument (Fig. 6). Use a tungsten target and a $0.3-\mathrm{mm}$ aluminum filter.

A tungsten target produces a higher quality, higher energy X-ray beam. Molybdenum is also a good target option and can be used for imaging materials with lower levels of mineralization.

A filter helps eliminate low-energy $X$-rays and minimize beam hardening artifacts. The exact thickness depends on the overall specimen and system configuration.

9. Manipulate the axes to bring the specimen into view. Visually check for air bubbles or metal particles that may have been missed.

Systematic Evaluation of

Skeletal

Mechanical

Function

Bubbles may be very light gray due to low attenuation of the X-ray beam. Metal particles appear sharp and black due to high attenuation of the beam.

10. Adjust the voltage and current settings as needed to optimize image quality, detail, and contrast. 


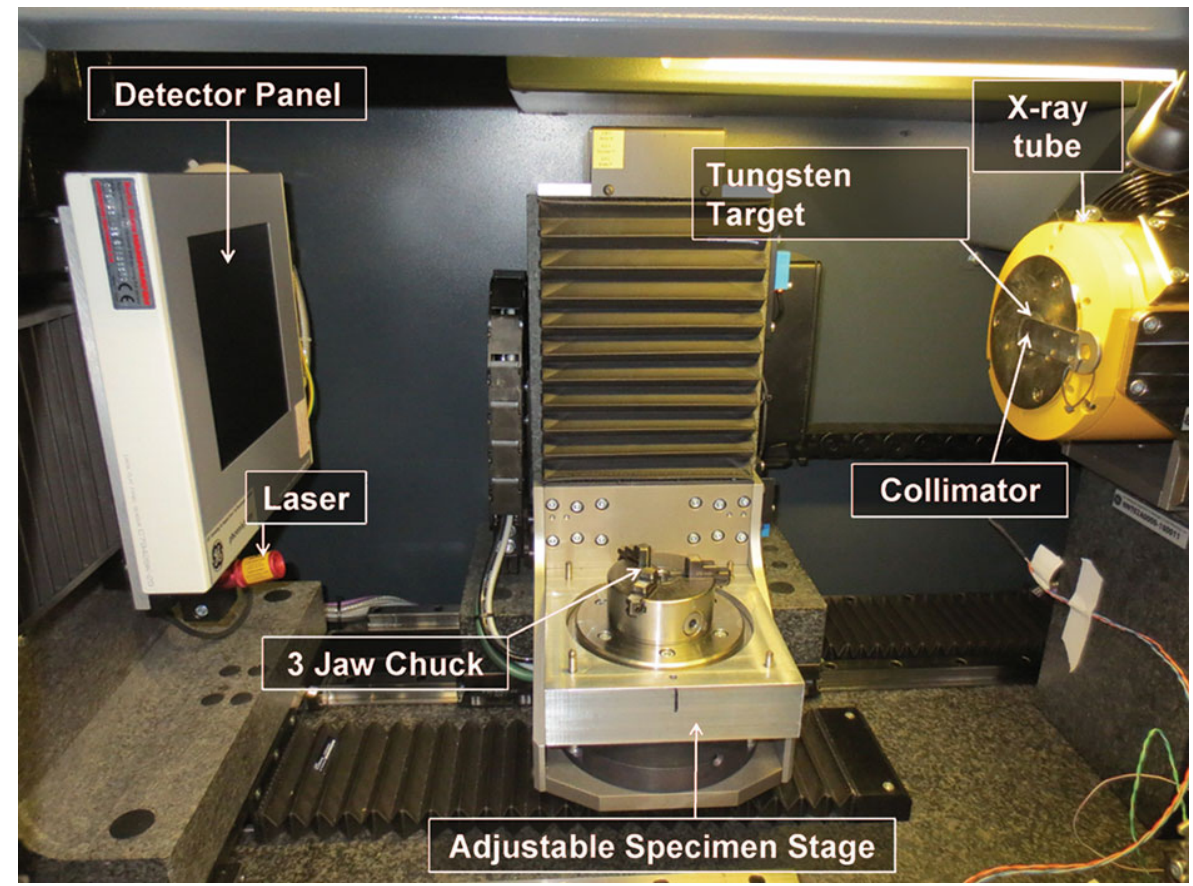

Figure 6 Inside cabinet of Nanotom S. Cylindrical tube fits securely into the three-jaw chuck between the X-ray source (right) and detector panel (left). In this system, the tube is stationary, the detector can shift to the right or left to allow a larger field of view, and the specimen rotates on the stage in the center. The laser is used to center the specimen between the tube and detector.

The ideal settings will depend on the specimen, solution, and holder. Higher voltages increase X-ray penetration power and are useful when the sample is dense or thick. However, increasing this value too much for a sample that is not very dense or thick will decrease image quality substantially, because the beam may pass right through the bone without being attenuated. For our system, a setting of $85 \mathrm{kV}$ works best.

Higher current settings are used to increase the number of X-rays reaching the sample, and therefore exiting the sample and reaching the detector. The result is a wider range of gray values and greater detection of detail. However, increasing this setting too much will widen the background peak, decreasing the signal-to-noise ratio. Furthermore, since radiation deposition into a sample is highly correlated with current, increasing the current increases the amount of radiation that the specimen will receive. For our system, a setting of $220 \mu \mathrm{A}$ works best.

It is critical to watch the overall power settings and stay within the acceptable power range of the system. Ignoring the system's maximum power settings will greatly decrease the overall lifetime of the system and its components.

11. Adjust the exposure time.

Increasing the timing increases the exposure of the detector to the X-ray beam for each image, thus increasing image quality. However, increasing timing also comes at a cost, as it significantly increases the scan length. A setting of $2000 \mathrm{msec}$ is best for decreasing graininess or noise without compromising scan time.

12. Select a suitable averaging value that will maximize image quality without compromising scan time.

Averaging specifies the number of images that will be taken at each angle and integrated to improve the signal-to-noise ratio. Higher increases scanning time substantially. Averaging three images is generally adequate.

Systematic Evaluation of

Skeletal

Mechanical

Function

49

Volume 3 
13. Adjust the number of skips.

This is the number of images per angle on the detector that are not recorded. Images are skipped to minimize the effects of detector afterglow. Skipping one frame is generally adequate; skips greater than one are generally used for very long, high-power scans. Again, this requires longer scan times.

14. Establish an observation region of interest (ROI).

This is a further correction of background signal. When choosing the representative ROI, ensure that no part of the sample passes through the region during the scan.

\section{Run scans}

15. Calibrate by running bright- and dark-field (gain and offset) images using the scanning parameters.

After positioning the sample to be scanned and optimizing the scanning parameters, the sample must be removed (holder and all) to calibrate the detector. Replace sample holder with a tube of water if the sample is being scanned in water. If the sample is being scanned dry, calibrate with nothing in the way of the beam.

Turn the cabinet light off during calibration and during the scan. The detector in the Nanotom $S$ model can detect the cabinet light, resulting in slightly shifted gray values. If using another system that has an inside cabinet light, verify with the system manufacturer or perform an onsite validation study.

16. Replace the sample and start the scan.

All six wells in the sample holder will be scanned (five bones and the calibration standard).

\section{Perform reconstruction}

Different reconstruction software programs are available. The following describes a general protocol that can be applied to most reconstruction programs in a stepwise fashion.

17. Select the region to reconstruct. If the specimen(s) do not take up the entire volume, it is best to select a sub-volume to decrease the reconstruction file size, which can be very large using nanoCT.

18. Correct for center of rotation errors in the scan.

19. Compare the first and last image to verify that there is no visible movement during the scan.

If there was no movement, the difference between the first and last images should be uniformly gray.

If there was movement, correct by shifting the $x$ and $y$ pixels. If the movement is uncorrectable, it will be necessary to reposition the sample and repeat the scan.

If there was movement of air bubbles or packing material around the sample, but no movement of the sample, do not correct the shift. Correction for movement in background will apply an unwanted shift to the specimen, resulting in decreased image quality of the specimen. Furthermore, air bubble movement during a scan is extremely difficult to correct.

20. Select to perform a median filter, ROI-CT filter and to utilize the observation ROI to correct for variation in background gray values that occur during the scan.

Some systems do not allow the selection of an observation ROI.

Systematic Evaluation of Skeletal

Mechanical Function
21. Save the reconstructed .vgi file.

22. Repeat the above procedure to reconstruct individual volumes of the calibration standards (i.e., air, water, and HA). 


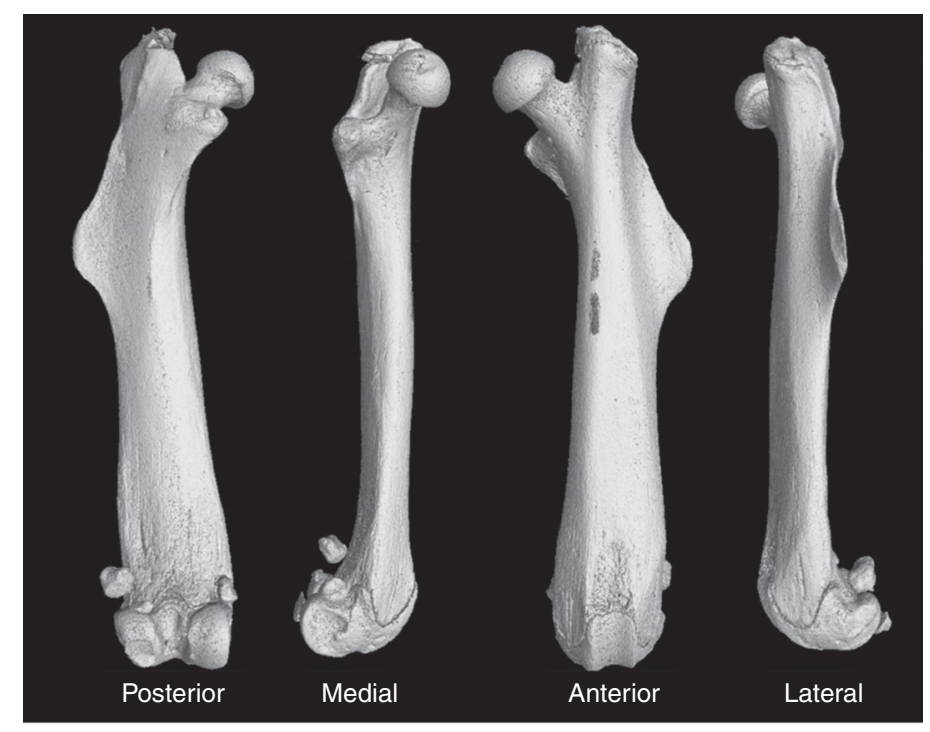

Figure 7 Iso-surface rendering of a single 16-week chromosome substitution strain male femur using VGStudio MAX 2.1 scaled in Hounsfield.

\section{Scale to Hounsfield units $(\mathrm{HU})$ and isolate individual bone volumes}

This protocol has been established specifically for use with VGStudio MAX 2.1 software, provided with the Nanotom S scanning system.

23. Import the .vgi file of interest.

24. Record the file dimensions ( $x, y$, and $z)$.

These will be necessary to import the image into MicroView for analysis.

25. Change the file type to signed 16-bit.

26. Select the histogram button, then the calibrate tab. Convert the gray values from the acquired image to $\mathrm{HU}$ values by entering the gray value from the air standard into the background box and mapping that to -1000.0 . Then enter the gray value of the water standard in the material box and map that to 0.0. Apply these changes.

When choosing the regions of standard to calculate the average gray value, be sure the region is not affected by artifacts (e.g., beam hardening, shadowing, bubbles).

27. Select the preview button. Isolate the single bone of interest (Fig. 7) by drawing an ROI using the four toggle tabs.

This is done to reduce overall file size and load time in the analysis software. The files generated are still very large.

28. Press the finish button.

The file will load in Volume Graphics.

29. Go to the file tab and export the file as a raw data set. Use the histogram in Volume Graphics to confirm that the file has scaled appropriately.

\section{Analyze images}

Import file into MicroView

30. Select the raw file.

31. Enter the $x, y$, and $z$ voxel size.

Systematic

Evaluation of

Skeletal

Mechanical

Function

51

Volume 3 
32. Select raw and 3D image boxes.

33. Change the file type to little endian and short.

34. Enter the $x, y$, and $z$ file dimensions.

\section{Analyze calibration standards}

35. Open the water volume. Select the largest possible ROI containing only water. Find the mean gray value for the volume selected and record this as the arbitrary density unit (ADU) for water.

36. Open the HA volume. Select a ROI that maximizes coverage of the sample but stays central enough to avoid any partial volume effects felt by the edges. Find the mean value for the volume and record this as the ADU for HA.

It is a good idea to analyze the HA for density and mean gray value as a way to verify that there is no drift in system scanning over time.

37. Reorient each bone volume so the long axis of the bone is parallel to the $z$ axis.

\section{Perform regional bone analysis}

For mid-shaft of femur

38a. Open the reoriented file.

$39 \mathrm{a}$. Using a cylindrical ROI, select a $2.5-\mathrm{mm}$ region of bone just below the third trochanter extending distally (Fig. 8A). Be sure to include both bone and non-bone regions.

This specific site is preferred because there is little trabecular bone that will be included during thresholding and it allows for consistency throughout analysis. Furthermore, this region is generally where the sample fails during whole-bone four-point bending tests (see Basic Protocol 4).

40a. Auto-threshold the region.

It is better to threshold samples individually, rather than apply a single threshold value for all samples. Variations in scanning parameters or even mineralization of the sample can affect the threshold value. A blanket threshold value could lead to decreased crosssectional area values for samples with lower mineralization, and increased values for samples with higher mineralization.

41a. Segment the cortical regions of the bone (Fig. 8B).

42a. Measure bone mineral content (BMC), bone mineral density (BMD), tissue mineral density (TMD), cortical area (Ct.Ar), moments of inertia $\left(I_{\max }, I_{\min }, J\right)$, cortical thickness (Ct.Th), marrow area (Ma.Ar), and total area (Tt.Ar).

For distal femur trabecular bone

38b. Load the reoriented file and perform a median filter if the image looks fuzzy.

$39 \mathrm{~b}$. Rotate the bone so that the distal portion is on top. Scroll through slices until just under the growth plate.

40b. Use the spline feature to create a ROI by outlining the trabecular bone, getting as close to the cortical shell as possible (Fig. 8C). Repeat for a fixed proximal distance, splining again every ten slices.

For any study, the length of the image volume should be based on a fixed distance (e.g., $2 \mathrm{~mm}$ ) or a percentage of bone length. This decision is based on acquiring volumes of trabecular bone that best represent the scientific question.

41b. Save the contours of this ROI, interpolate, and generate a 3D ROI. 

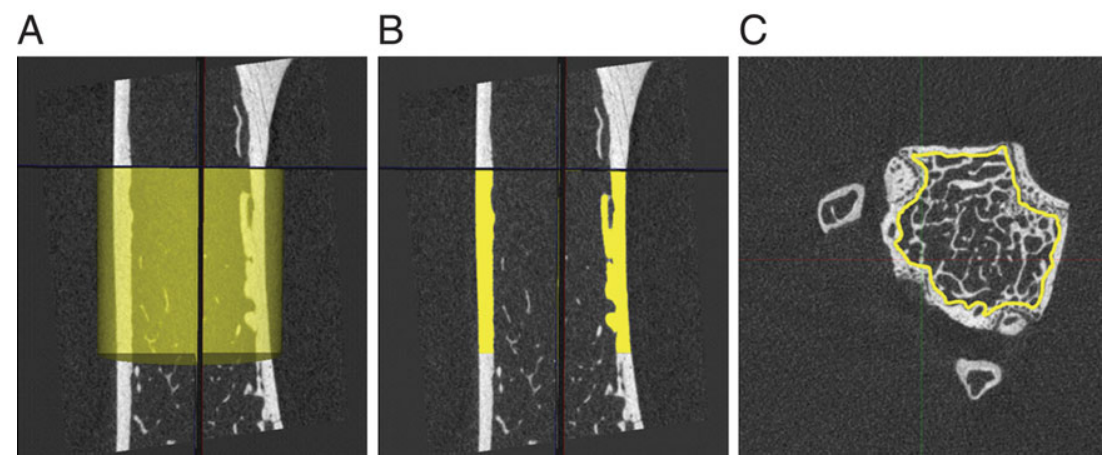

Figure 8 Images from MicroView Advanced Bone Analysis on a mouse femur. (A) Cylindrical region of interest (yellow) selected just below the third trochanter. (B) Selected region of interest with cortical bone segmented from trabeculae. (C) Distal femur slice just proximal to the growth plate. Yellow line shows spline separation of trabeculae and cortical shell.

42b. Auto-threshold the 3D ROI.

43b. Enter the HA ADU and the water ADU determined during the calibration standard analysis.

44b. Measure BMC, BMD, bone volume/total volume (BV/TV), connectivity of the trabecular structure, trabecular plate thickness, trabecular plate number, and trabecular plate separation.

\section{For vertebral trabecular bone}

38c. Load the reoriented file.

39c. Use the transverse view to analyze the image. Scroll to the most distal end of the vertebra, just past the growth plate.

40c. Select spline and outline the trabecular bone, getting as close to the cortical shell as possible, because the contours will also be used later for cortical analysis.

Be sure not to include any of the processes in the trabecular contours.

41c. Spline through each slice until the proximal growth plate is reached.

42c. Save the contours, then click interpolate and generate 3D ROI. Verify that the merging of the splines does not cut out any trabeculae or include any cortical bone.

43c. Save the cropped region containing only trabecular bone.

44c. Open the cropped trabecular file and apply a median filter if the image looks slightly fuzzy.

45c. Auto-threshold the image.

46c. Measure the same traits as for the distal femur (step 42a).

\section{For vertebral cortical bone}

38d. Perform as described for the mid-shaft femur (steps 38a-42a), but open the file and load the contours saved in step $42 \mathrm{c}$ of the vertebral trabecular bone analysis.

Systematic Evaluation of Skeletal

Mechanical

Function

53

Volume 3 
BASIC PROTOCOL 4
Systematic Evaluation of Skeletal Mechanical Function
MEASUREMENT OF WHOLE-BONE MECHANICAL PROPERTIES USING A FOUR-POINT BENDING TEST

Whole-bone four-point mechanical testing can be used to quantify mechanical properties of the cortical bone. These properties include stiffness, maximum load, post-yield deflection, and work-to-fracture. This protocol tests bones to failure, so if intact bones are needed for other analyses (i.e., imaging), be sure to complete those analyses first.

Several companies manufacture high-quality testing systems; each operates slightly differently in terms of software and hardware. The steps below are presented in general terms to allow for these differences. This protocol requires a mechanical testing system with an actuator travel of $\pm 5 \mathrm{~mm}$, a testing rate of $0.05 \mathrm{~mm} / \mathrm{sec}$, and a sampling frequency of $25 \mathrm{~Hz}$. You will need to record the test data, specifically the test time, displacement, and load. If your system does not include software for recording data, you may need to use another program (i.e., LabVIEW) to do so. Before conducting this protocol, it is important to be familiar with the operation of the testing system. You should be able to run a standard warm-up and change the testing procedure using either the software or the system's front panel, depending on your system.

The four-point testing fixture should have loading points that are a set distance apart. The two points on the bottom should be $6.5 \mathrm{~mm}$ apart, and the two points on top should be centered relative to the bottom points, and $2.2 \mathrm{~mm}$ apart. There must be some adjustment capability in the vertical direction of one loading point, so that variation in bone width can be taken into consideration. This ensures that all four loading points engage the bone at the beginning of the test. In some setups, the bottom points are adjustable in the vertical direction independent of each other; in other systems the two bottom points are machined to move as one unit. Adjustability as a unit or individually in the bottom two points is a matter of personal preference.

The protocol below details mechanical testing of mouse femora. It can also be used for mouse tibia and metatarsals, although the distances between the points may have to be modified, depending on bone length. Alternatively, the same bones can also be tested using a three-point fixture (see Alternate Protocol 1). For vertebral bodies, specialized fixtures are used to measure spine compression, which more accurately reflects normal spinal loads (see Alternate Protocol 2).

\section{Materials}

Whole frozen femurs (see Basic Protocol 1)

$1 \times$ phosphate-buffered saline $(\mathrm{PBS})$

Mechanical testing system with four-point fixture (Fig. 9A-C)

Absorbent pads

Gauze

Small-diameter $(\sim 2-\mathrm{mm})$ wooden dowel rods cut into 1-inch $(\sim 2.5$-cm) segments Forceps

Glass Petri dish

\section{Prepare for testing}

1. Remove bones from the freezer. Allow them to thaw $1 \mathrm{hr}$ before testing. Measure and record bone lengths.

Bones must be completely thawed before testing.

2. Warm up the mechanical testing system according to manufacturer's guidelines. We run our hydraulic system for $15 \mathrm{~min}$, cycling $\pm 5 \mathrm{~mm}$. 


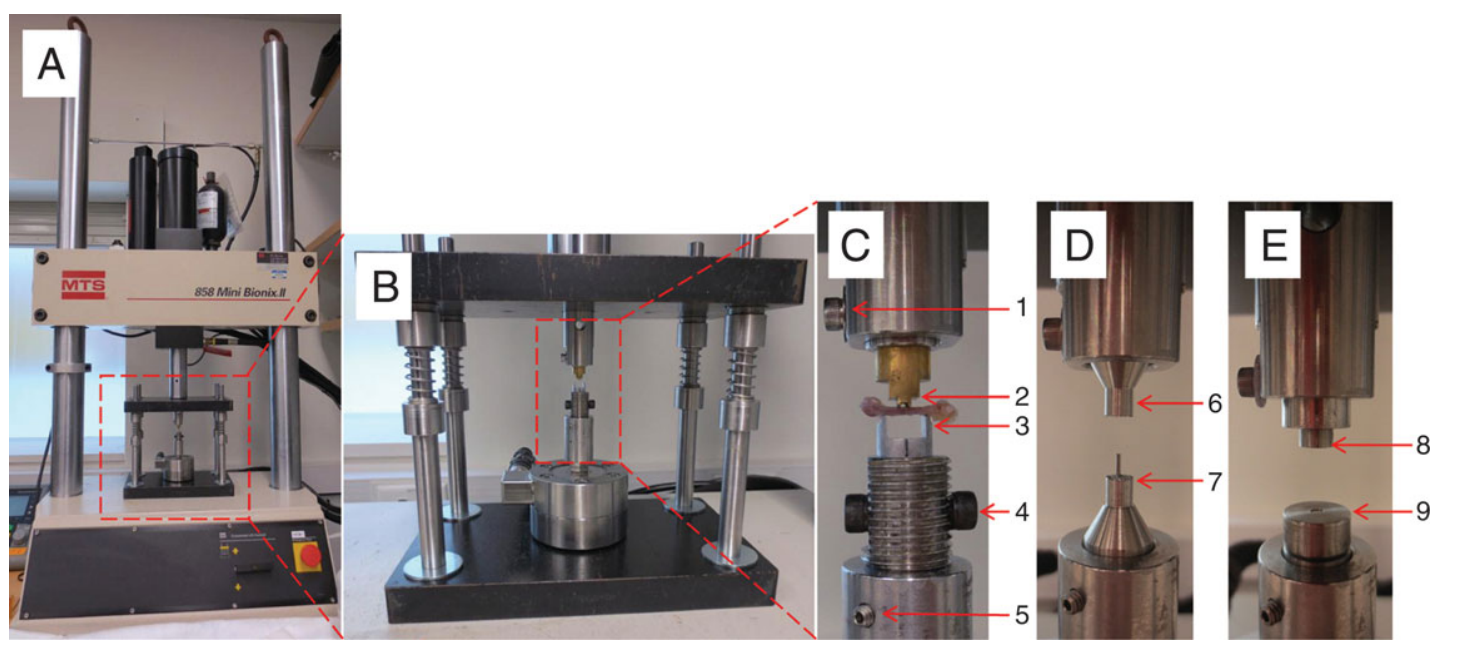

Figure 9 Mechanical testing setup used for whole-bone testing. (A) Entire system is shown with a small custom frame to hold testing fixtures. (B) Closer view of smaller frame on the testing machine. This is adjustable to incorporate different load cells and testing fixtures. (C) Four-point bending fixture with bone ready for testing. (1) Screw that adjusts the vertical height of the upper two points; (2) upper two points resting on the bone; (3) lower two points; (4) screws used to individually adjust height of the bottom points; (5) additional screw for adjusting lower points, with the ability to rotate them and lock them into place. (D) Compression testing fixture for lumbar vertebrae. $(6,7)$ Upper and lower platens. Latter has a peg to help position the vertebra. (E) Compression testing fixture for caudal vertebrae. $(8,9)$ Top and bottom platens have a small depression in the middle to help position the vertebra. Bottom platen rests on a peg so that it can be tilted slightly, if needed, to align the vertebra.

3. While the system is warming up, prepare the testing area. Place an absorbent pad next to the testing system where the bones will be prepared. On the absorbant pad, place gauze, wooden dowel rod sections, forceps, a bottle of PBS, and a glass Petri dish containing PBS.

It is important to have a clear strategy for knowing which bones have been tested and which have not. Preparing two separate areas or containers generally works best.

4. After system warm-up is complete, set the system so the test will run at a displacement rate of $0.05 \mathrm{~mm} / \mathrm{sec}$ and data will be recorded at $25 \mathrm{~Hz}$. If able, set a distance limit so that there is no danger of yielding a load cell if the bone does not break.

\section{Test standards}

5. Place a 12-mm section of wooden dowel in the four-point testing fixture.

6. Adjust the top two points so they just contact the dowel rod without applying a load.

7. If needed, adjust the fixture so that both bottom points are in contact with the dowel rod, again without applying a load.

For a set-up in which one of the bottom points is adjustable in height, leave one point set and adjust the other slightly, as needed, to ensure that all four loading points touch the sample prior to testing.

8. Begin the test and record load and deflection data. Pay close attention to the testing fixtures, so that the actuator can be stopped as soon as the dowel rod breaks.

Do not let the top of the testing fixture come into contact with the bottom of the testing fixture! This will yield the load cell, causing permanent damage. This can be avoided by building an emergency stop button into the software or by using the system's physical emergency stop button.

Systematic Evaluation of Skeletal

Mechanical

Function

\section{5}

Volume 3 
In our testing setup, a program loads the specimen when a start button is clicked. It automatically loads the specimen at a set rate, records the data, and displays real-time load-deflection results on-screen. A reset button, if pressed at any point during the test, will automatically bring the testing system back to the starting position.

9. Bring the actuator back to the starting point.

10. Repeat with enough dowel rods to ensure confidence in positioning and comfort in running the testing system.

\section{Test bones}

11. Remove a bone from its tube and place it in the Petri dish containing PBS.

Keep bones hydrated at all times. If a bone has been out of PBS for more than a few seconds because the test has been delayed for any reason, return the bone to the Petri dish and rehydrate. Dehydration will make bones brittle, and will be reflected in reduced post-yield deflection and work-to-fracture.

12. Using gauze, remove any residual soft tissue from the bone and return to the Petri dish.

13. Record which bone is being tested.

14. Place the bone in the four-point bending fixture with the anterior side facing down and the distal end to the left (Fig. 9C). Make sure the bone is centered in the testing fixture from front to back.

Testing in the posterior-to-anterior direction is preferred because it subjects the anterior side of the bone to the tensile loads. Although the anterior and posterior sides are both comprised of lamellar tissue, the organization of the anterior side seems to handle tensile loads better than the posterior side, so that posterior-to-anterior loading results in greater post-yield deflection compared to anterior-to-posterior loading. The stiffness and maximum load are essentially the same for the two loading directions.

15. Adjust the loading points, if needed, to ensure that all four points are in contact with the bone.

16. Load the bone at a rate of $0.05 \mathrm{~mm} / \mathrm{sec}$ until it breaks.

Usually there will be a distinct popping noise when the bone breaks. If load and displacement data can be monitored during the test, there will also be a significant drop in load. Depending on the mouse being tested, some bones will deflect significantly before breaking. If the bone continues to bend without breaking, stop the test when the load decreases to $<50 \%$ of the maximum load.

The displacement rate may affect whole-bone mechanical properties, particularly postyield deflection. In general, post-yield deflection is inversely related to displacement rate. A brittle bone generally shows low post-yield deflection no matter how fast it is loaded. A normal bone will show low post-yield deflection at faster loading rates. If it is suspected that a perturbation may make the bone more brittle, use the $0.05 \mathrm{~mm} / \mathrm{sec}$ displacement rate to exaggerate differences between control and experimental groups.

17. Record the location of the break along with any abnormalities that may have occurred during the test or any oddities about the bone tested.

Entries could look like "Test1. B6 mouse1 broke in middle".

In four-point bending, the bone can break in the middle or on the right or left side. Because of the design of the testing fixtures, the bone can break anywhere between the two upper points.

Systematic Evaluation of Skeletal Mechanical Function 56

18. Place the broken bone back in the tube and place the tube in the area designated for bones that have been tested.

19. Repeat until all the bones have been tested. 


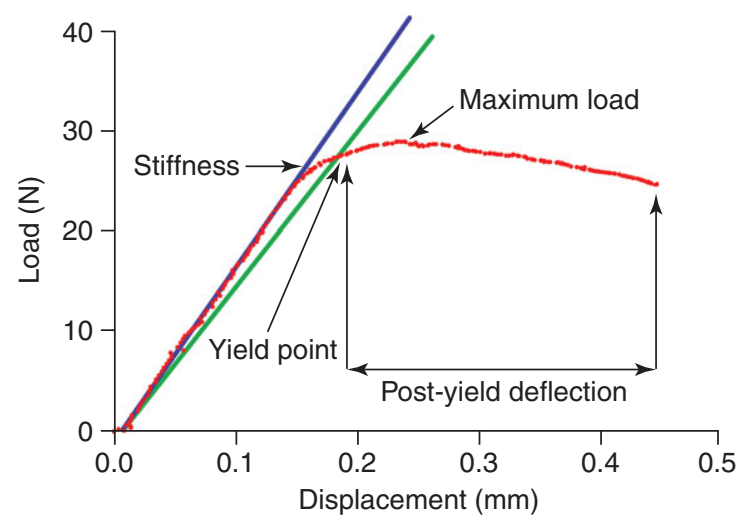

Figure 10 Typical load-displacement curve. Stiffness is indicated by the blue line. The green line is stiffness multiplied by 0.9 , which is useful when calculating yield point.

\section{Analyze data}

20. Use Excel or a custom analysis program in a software package such as MatLab or LabView to determine the following whole-bone mechanical properties (Fig. 10):

Stiffness. A measure of how much a bone deforms under a given load. For example, a steel rod is stiffer (deforms less under load) than an aluminum rod.

Maximum load. The highest load a bone can sustain prior to fracturing. In whole-bone mechanical tests, it is convention to use the term maximum load and not strength, which is often used incorrectly. Strength is similar to maximum load in concept (i.e., greatest load sustained), but is used to describe tissue-level mechanical properties, when the effects of geometry have been taken into consideration.

Yield point. The point at which a loaded structure undergoes permanent change, such as accumulation of damage. The structure is now more compliant (less stiff), and this is reflected on a load-deflection graph as a bending in the curve. Because the curves generally show a gentle change in slope, one simple way to assess the yield point in a standardized way is to multiply the stiffness by 0.9 and then find the point on the curve where this degraded stiffness intersects. There is no hard-and-fast rule to define yielding, and it is up to the investigator to standardize this as he or she sees fit.

Post-yield deflection. The amount of deflection a structure undergoes after sustaining damage (i.e., yielding) but before failing. A brittle material will show very little post-yield deflection prior to failing, whereas a ductile material will show a rather large deflection prior to failing.

Work-to-fracture. Calculated as the area under the load-deflection graph. This property is often difficult to interpret, given that the area under the graph depends on both the height of the curve (i.e., maximum load) and width of the curve (i.e., post-yield deflection). If a mutation is associated with a reduction in work-to-fracture, the first thing to do is establish whether there was a change in maximum load and/or post-yield deflection. This is important because the skeletal changes leading to reduced maximum load are very different from those leading to reduced post-yield deflection.

21. Determine the bending stiffness (EI) as follows:

$$
\mathrm{EI}=(P / y)\left(a^{3} / 3-a^{2} L / 4\right)
$$

Systematic Evaluation of Skeletal

Mechanical Function

57

Volume 3 
ALTERNATE PROTOCOL 1

ALTERNATE PROTOCOL 2

Systematic Evaluation of Skeletal Mechanical Function where $P / y$ is stiffness (from the load-deflection curve), $L$ is the span length of the lower two supports, and $a$ is the span of the upper loading points.

Bending stiffness is an important property that adjusts for the test fixture geometry. The equation takes into account the load, deflection, and geometry of the test setup.

\section{MEASUREMENT OF WHOLE-BONE MECHANICAL PROPERTIES USING A THREE-POINT BENDING TEST}

Mechanical testing with a three-point testing fixture utilizes a fixture that holds the bone at only three loading points: one upper point positioned between two lower points. The test is similar to testing with a four-point testing fixture; loading rate and sampling frequency are the same. The biggest difference is where the bone will break. In a threepoint fixture, the bone will break in the middle, where the single upper loading point comes into contact with the bone. For four-point bending, the bone can break anywhere between the top two points. Choosing between systems with a three-point fixture versus a four-point fixture is really a matter of personal preference. Unpublished data from our lab shows no significant difference between testing with three- or four-point fixtures. Part of the decision may come down to the machining capabilities available for designing and building the fixtures. Four-point bending may be preferable as it allows the bone to break in the mid-shaft where it is weakest. In three-point bending, the user decides where the bone will break along the diaphysis. When testing with a three-point fixture, the procedure is the same as with a four-point bending fixture, except for the adjustment of the loading points. Start by making sure the two bottom points are in contact with the bone, then adjust the top (third) point so that is in contact with the bone.

\section{MEASUREMENT OF SPINE COMPRESSION}

For vertebral bodies, compression testing is useful because it is consistent with the types of loads expected during habitual loading. Unlike long bones, vertebral bodies contain both cortical and trabecular bone, and both tissue types determine overall strength. Both lumbar and caudal vertebrae work for this method. Caudal vertebrae are a bit easier to work with, but are more difficult to interpret in the context of the human skeleton. In this procedure, the loading rate can be modified as needed to be consistent with prior work.

\section{Additional Materials (also see Basic Protocol 4)}

Whole frozen vertebrae (see Basic Protocol 1)

Appropriate standard, e.g., pencil-top erasers

Lumbar or caudal testing fixtures (Fig. 9D,E)

\section{Prepare for testing}

1. Thaw bones and prepare the test area as described (see Basic Protocol 4, steps 1 to 4$)$.

2. Once the machine is warmed up, position the lumbar or caudal testing fixture. Do as much pre-adjustment as possible.

The testing fixtures should line up vertically so that the vertebra will experience an axial load.

Some fixtures have a wire that can be used to center the fixtures before the testing platens are in place. If there is no such set-up, check the alignment by very slowly pushing the fixtures together by hand to ensure they are aligned vertically.

\section{Test standards}

3. Position the standard in the fixture the same way the bone will be positioned. Make sure the standard is straight in the vertical direction to ensure an axial load. Move 
the system actuator so that it holds the standard in place without putting any load on it.

There is a fine balance in determining this point, which is why it is best to test the system and fixtures using easily replaceable standards, not important bone specimens. A suitable standard should be easily accessible and plentiful, so that the same type of standard can be used to check the system and setup over the course of an entire study. Pencil-top erasers work well.

4. Run the test using a $25 \mathrm{~Hz}$ sampling rate and $0.05 \mathrm{~mm} / \mathrm{sec}$ displacement rate. Make sure the system is recording the load, displacement, and time of each test.

5. If able, set a limit on the actuator distance. Make sure the limit will allow the bones to be tested, but will prevent the testing platens from making contact and damaging the load cell.

6. Run several tests to ensure confidence that the testing setup is working properly.

\section{Test vertebra}

7. If the spine was harvested in sections, disarticulate a single vertebra once the section has thawed.

Keep bones hydrated at all times. If a bone has been out of PBS for more than a few seconds because the test has been delayed for any reason, return the bone to the Petri dish and rehydrate. Dehydration will make bones brittle, and will be reflected in reduced post-yield deflection and work-to-fracture.

8. Use gauze to remove as much soft tissue from the vertebra as possible for easier positioning. Do not damage the bone sample while removing the soft tissue.

9. Position the bone in the appropriate fixture:

a. For lumbar spine testing, place the vertebra so that the peg of the fixture goes into the vertebral foramen, with the spinous process facing the back of the machine. Bring the top platen down very slowly so that it touches the vertebra without loading it.

b. For caudal spine testing, place the vertebra so that the distal end is resting in the slight indentation of the bottom platen and the proximal end fits into the slight indentation in the top platen. Bring the top platen down very slowly so that it touches the vertebra without loading it.

10. Run the test as in steps 4 and 5 .

11. Repeat for all bone samples.

\section{Analyze data}

12. Plot a load-displacement curve using units of $\mathrm{N}$ versus $\mathrm{mm}$ for easiest comparison to the literature.

13. Determine stiffness from the slope of the curve.

14. Determine the maximum load.

\section{MEASUREMENT OF TISSUE-LEVEL MECHANICAL PROPERTIES}

Changes in tissue-level mechanical properties can be measured directly in mouse bones, despite their small size. Most studies estimate tissue modulus from whole-bone bending tests using engineering beam theory. This method should be used with caution. First, the estimates are generally in the 5-15 GPa range. Mouse bone has a tissue modulus of 25-35 $\mathrm{GPa}$. Second, tissue modulus estimated from whole-bone bending tests does not correlate

BASIC

PROTOCOL 5

Systematic Evaluation of Skeletal

Mechanical

Function

\section{9}

Volume 3 
well with that measured from direct mechanical testing. Third, only tissue modulus and tissue strength can be estimated from whole-bone bending tests. Estimating tissue modulus may be sufficient if the experimental and control groups have very different tissue-level mechanical properties; however, if tissue-level mechanical properties are similar and if the perturbation is expected to alter tissue toughness, it is best to measure tissue-level mechanical properties directly.

\section{Materials}

Fresh or frozen bone samples, e.g., femurs (see Basic Protocol 1)

Scotch-Weld acrylic adhesive DP-810

Basic fuchsin

Caroplastic (Carolina Biological Supply Co.)

Alignment device

Brass pots

CNC milling machine (Modela MDX-20, Roland DGA)

Light microscope (e.g., Zeiss Axioplan2, Carl Zeiss IMT)

Micro-scissors

Four-point bending fixture (see Basic Protocol 4)

Servo-hydraulic materials testing system (Instron model 8872)

High-resolution digital video camera (RH1100, Duncan Tech)

Video zoom microscope lens (Edmund Industrial Optics)

16- $\mu \mathrm{m}$ silicon carbide particles (McMaster-Carr, optional)

Low-speed diamond-coated wafering saw (Buehler)

Acrylic slides

Digital Exwave HAD 3CCD Camera (Sony)

Digital-image correlation (IMAQ Vision Builder 6.0, National Instruments Corp.)

\section{Prepare samples}

1. Clean the sample.

2. Embed both metaphyses in brass pots with Scotch-Weld acrylic adhesive DP-810. Use an alignment device to ensure the brass pots are aligned.

3. Machine the medial and lateral sides of the potted bone samples to generate an anterior and posterior spicule connecting the proximal and distal halves of the femur.

An inexpensive automated CNC milling machine can be used. The gauge lengths of the spicules should be $\sim 3 \mathrm{~mm}$ long with widths of $280 \mu \mathrm{m}$. All machining should be conducted under constant water (PBS) irrigation.

The periosteal and endosteal surfaces of each specimen should not be machined in order to minimize microstructural defects due to machining.

4. Under a microscope, clip out samples and load in a four-point bending fixture.

Alternatively, samples can be loaded with tension at $10 \mu \mathrm{m} / \mathrm{sec}$ using a servo-hydraulic materials testing system.

Minimize bending loads during the test by precisely aligning all test fixtures using a micrometer stage.

\section{Take measurements}

5. Measure deformations using a high-resolution digital video camera and a video zoom microscope lens. To enhance optical tracking of deformation, "pepper" the sample with $16-\mu \mathrm{m}$ silicon carbide particles.

Systematic Evaluation of Skeletal

Mechanical Function

6. Calculate strain values using digital-image correlation by tracking the movement of the silicon carbide particles. 
7. After the sample has been loaded to failure, retrieve the failed ends to measure the cross-sectional area.

8. Stain the fracture sample with basic fuchsin (e.g., Jepsen et al., 1999).

9. Embed the sample in Caroplastic according to manufacturer's instructions.

10. Section the sample transversely (200 $\mu \mathrm{m}$ thickness) using a low-speed diamondcoated wafering saw.

11. Fix sections to acrylic slides using cyanoacrylate, and polish to a 1- $\mu \mathrm{m}$ finish using a series of sandpapers followed by diamond suspension slurries.

12. Image the sample with a digital Exwave HAD 3CCD camera and a light microscope.

13. Measure cross-sectional area for three to six sections per sample and average the values.

\section{Analyze data}

14. Divide all force data by the cross-sectional area to calculate stress. Plot stress against the strain determined from the optical tracking system.

15. From the stress/strain curves, calculate strength, post-yield strain, work-to-failure, and Young's modulus. Calculate modulus values from the initial, linear portion of the curve before yield. Yield can be defined in the traditional sense using the $0.2 \%$ offset method.

The overall accuracy of the mechanical testing and imaging methods can be verified using milled aluminum, which has a known elastic modulus of $70 \mathrm{GPa}$.

\section{MEASUREMENT OF ASH WEIGHT, WATER CONTENT, AND BONE DENSITY}

Assessing ash content is a relatively simple first step toward assessing changes in matrix composition and provides a measure of the degree of mineralization. Additional tests using Raman or Fourier-transform infrared (FTIR) imaging can be conducted to generate more refined information about changes in mineral and collagen content. Variations in mineralization are small (on the order of $2 \%-4 \%$ ), but can result in rather large differences in tissue-level mechanical properties. Thus, establishing a consistent method for weighing and ashing is important to obtain meaningful results. Each lab should verify the times listed below so that weight measurements represent the steady-state value.

The following ash weight protocol is appropriate for cortical bone specimens (murine or cadaveric) with an overall specimen weight $>8-10 \mathrm{mg}$ and with very little fat. It should not be used with specimens that have closed medullary canals. However, it has been validated that there is no difference between defatted and non-defatted densities for mouse bone (Jepsen, unpub. observ.).

It is important to include a standard that can be tested over time using the same protocol so that the system gives consistent results without drift. An aluminum ring is a great option, because it has known properties (density $=2.7 \mathrm{~g} / \mathrm{cc}$ ) and is easily obtainable.

\section{Materials}

Fresh or frozen bone samples (see Basic Protocol 1) or broken bones from four-point bending tests (see Basic Protocol 4)

Distilled water

Stereomicroscope

Vacuum oven (Fisher Scientific Isotemp or Eurotherm 91e Blue)

Systematic Evaluation of Skeletal

Mechanical

Function

\section{1}

Volume 3 
Analytical balance with strain gauge wire (Fig. 11)

Delicate task wipes

2-ml microcentrifuge tubes

Microcentrifuge (Fisher Scientific accuSpin Micro 17)

Ashing oven (Thermo Lindberg Blue M)

\section{Measure submerged weight}

1. Remove all soft tissue (e.g., periosteal tissue, marrow) under a stereomicroscope. Cut off distal and proximal ends, leaving a diaphaseal region for the rest of the steps.

2. Submerge specimens in distilled water. Degas by placing the submerged specimens in a 5- to 7-mmHg vacuum for $2 \mathrm{hr}$.

3. Remove from vacuum and let stand for $1 \mathrm{hr}$ at atmospheric conditions.

4. Measure submerged weight in distilled water by hanging the specimen from a fine wire (Fig. 11) and record the weight of the specimen suspended in water.

\section{Measure hydrated weight in air}

5. Place a delicate task wipe at the bottom of a 2-ml microcentrifuge tube.

6. Place the specimen in the tube and close the cap to control humidity.

7. Centrifuge $10 \mathrm{~min}$ at $8000 \times g$.

8. Weigh the specimen.

Weigh only four to five specimens at a time to minimize errors from drying.

\section{Measure dried weight}

9. Place specimen in an $80^{\circ} \mathrm{C}$ vacuum oven for at least 3-5 hr (overnight is optimal).

10. Remove from oven and weigh in air.

Systematic Evaluation of Skeletal

Mechanical Function

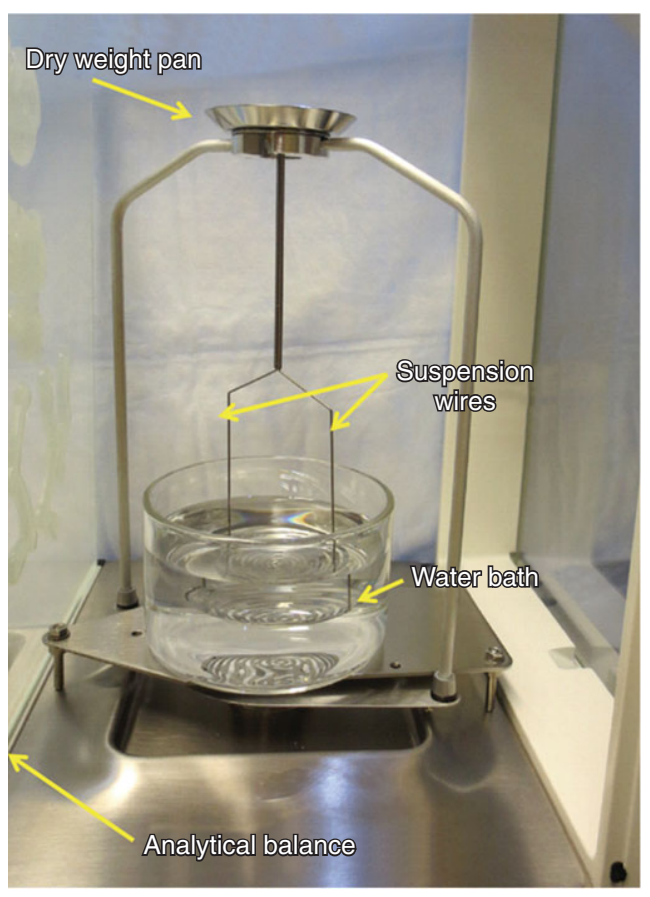

Figure 11 Density kit for use with an analytical balance. This kit contains a pan to measure dry weight as well a water bath and suspension wires to measure wet weight. 


\section{Measure ash weight}

11. Place specimen on a ceramic plate or crucible and leave in a $600^{\circ} \mathrm{C}$ ashing oven for $18 \mathrm{hr}$.

12. Remove from oven and weigh in air.

13. Determine submerged weight of ash (see steps 2 to 4 ).

\section{Perform calculations}

14. Determine the following values for volume, density, and content:

Hydrated bone volume $=$ hydrated weight in air - submerged weight

Dry bone volume $=$ dry weight in air - submerged weight

Ash volume $=$ ash weight in air - submerged ash weight

Hydrated bone density = hydrated weight in air/hydrated bone volume

Dry bone density $=$ dry weight in air/dry bone volume

Ash density $=$ ash weight in air/ash volume

Water content $=$ (hydrated weight in air - dried weight $) /$ hydrated weight

Ash content $=$ ash weight/hydrated weight or ash weight/dry weight

Organic + carbon dioxide content $=($ dry weight - ash weight $) /$ hydrated weight .

\section{SYSTEMATIC DATA ANALYSIS}

The following protocol provides an approach to assessing functional and morphological differences between knock-out (KO) and wild-type (WT) mice.

\section{Perform functional analysis}

1. Test whether the mutation affects the following parameters:

bone stiffness

maximum load

post-yield deflection

work-to-fracture.

For KO and WT mice, regress each parameter against body mass or body mass $x$ length (e.g., see Fig. 12A for bone stiffness). Test for between-group differences in slope and $y$-intercept for each parameter using analysis of covariance (ANCOVA).

Post-yield deflection does not typically correlate with body mass or body mass $\times$ length.

Alternatively, use a general linear model to evaluate the above functional characteristics for each group, using body mass or body mass $\times$ length as a covariant.

If KO and WT groups do not show large differences in body mass, it is acceptable to test for differences in the mechanical properties using a t-test. It is highly advised to first check how each mechanical property varies with body mass to confirm that the KO did not affect the relationship between mechanical properties and body mass that may not be predicted based an analysis of group means alone (e.g., a KO may not show a difference in stiffness compared to WT, but may show a different relationship between stiffness and body mass, as determined based on ANCOVA).

\section{Perform morphological analysis}

2. Use the same regression strategy to test whether the mutation affects on the following parameters:

Systematic Evaluation of Skeletal

Mechanical

Function

63

Volume 3
BASIC PROTOCOL 7 

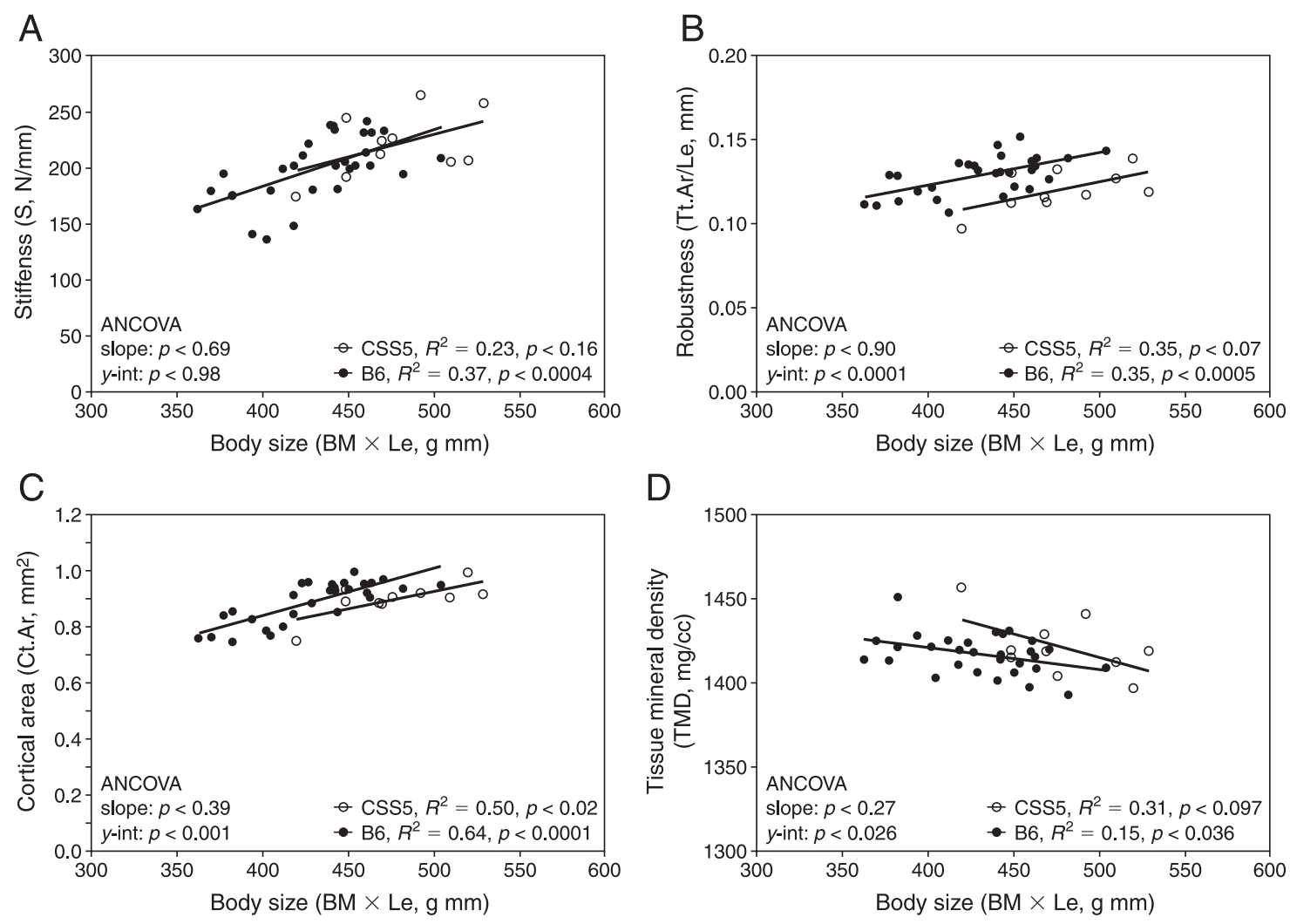

Figure 12 ANCOVAs of body size (body mass $\times$ length) and various traits for chromosome substitution strain 5 (CSS5) and control (B6) mice at 16 weeks. (A) Stiffness, (B) robustness, (C) cortical area, and (D) tissue mineral density (TMD). $p<0.008$ is considered significant.

Systematic Evaluation of Skeletal Mechanical Function
Regress total area (Tt.Ar) to assess periosteal expansion Regress robustness (Tt.Ar/Le) to assess the relationship between periosteal expansion and growth in bone length (Fig. 12B)

Regress marrow area (Ma.Ar) to assess marrow expansion Regress cortical area (Ct.Ar) to assess accrual of bone mass (Fig. 12C) Regress tissue mineral density (TMD; Fig. 12D) and ash content.

For KO and WT mice, regress each parameter against body mass or body mass $\times$ length and test for between-group differences in slope and $y$-intercept using ANCOVA.

Typically, TMD is not highly correlated with body mass or body mass $\times$ length.

Alternatively, generate a general linear model with each of the above traits to compare KO and WT, using body mass or body mass $\times$ length as a covariant.

\section{For vertebral bodies}

3. Analyze the cortical shell as described for the femoral diaphysis (see Basic Protocol 3, steps 38a-42a) to assess how the mutation affects the bone volume fraction (BV/TV).

4. Determine how trabecular thickness and trabecular spacing vary with body mass or body mass $\times$ length as factors contributing to stiffness (see step 1 above).

5. If a change in BV/TV is found in a region, also analyze the cortical area around that region for subsequent changes. 
Table 1 Expected Changes in Functional, Morphological, and Compositional Traits

\begin{tabular}{lllll}
\hline Robustness & Ct.Ar & TMD & Stiffness & Maximum load \\
\hline Slender & Reduced & Increased & No change & No change \\
Slender & Very reduced & No change & Reduced & Reduced \\
No change & Reduced & No change & Reduced & Reduced \\
No change & Increased & Increased & Increased & Increased \\
Increased & Increased & Reduced & No change & No change \\
Increased & Increased & No change & Increased & Increased \\
\hline
\end{tabular}

The variation in BV/TV should be largely explained by trabecular thickness (Tb.Th) and trabecular number (Tb.N). This should be checked to confirm that scanning was conducted properly. This check also provides insight into the underlying biological factors contributing to variations in BV/TV. Variations in Tb.N may result from excessive resorption near the growth plate or in the metaphyseal region. Variations in Tb.Th may indicate an imbalance between osteoblastic formation and osteoclastic resorption.

Mouse bone is extremely adaptive. If the KO resulted in a more slender phenotype, this would be associated with reduced Ct.Ar relative to body size and increased TMD. Not seeing these additional changes would likely also be associated with differences in stiffness and maximum load relative to body mass. There are no norms specifying the exact amount of change in Ct.Ar and TMD expected for a slender phenotype. At this point, it is sufficient to interpret the changes in the context of whole-bone mechanical properties. Variations in whole-bone mechanical properties are best interpreted in the context of how multiple traits are affected by the mutation. Table 1 presents several scenarios for how a KO may alter structural and material properties and the subsequent effects on whole-bone mechanical properties.

\section{COMMENTARY}

\section{Background Information}

Most physiological systems are considered complex adaptive systems because they are capable of coordinating multiple traits simultaneously in order to generate organlevel function or homeostasis (Wright, 1921; Waddington, 1942; Nadeau et al., 2003; Marder and Goaillard, 2006; Jepsen, 2009). In the skeletal system, a key requirement of bone is to be sufficiently stiff and strong to support loads imposed during habitual loading. As the skeletal system is adaptive, genetic and environmental perturbations affecting one bone trait are often accompanied by compensatory changes in other traits (Jepsen et al., 2007, 2009, 2010). The challenge is to figure out which alterations can be attributed to the perturbation and which result from adaptive changes associated with establishing mechanical function. For example, early research in transgenic mouse models revealed that a mutation affecting type I collagen gene expression led to an age-related increase in bone size (Bonadio et al., 1993). This adaptive response occurred presumably to offset the reduced tissue stiffness associated with the type I collagen mutation (Jepsen et al., 1997). If the mice were assessed at a single adult age, one might conclude that producing less collagen makes a bigger bone. This statement is not entirely incorrect, but it is certainly misleading, particularly in the context of potential treatment strategies for osteoporosis.

A noteworthy example of how a genetic mutation can affect bone morphology but not bone strength was presented by Maloul et al. (2006). Gdf7 (BMP-12), which is typically thought to regulate soft-tissue healing (Lou et al., 2001), has also been shown to affect proliferation and phenotypic expression of osteoblastic (Ros 17/2.8) cells in culture (Furuya et al., 1999). Interestingly, the $G d f 7$ deficiency impaired sub-periosteal expansion of femora, leading to a slender (narrow) phenotype, but did not affect bone strength. Thus, the skeletal system in these mutant mice had altered tissuelevel mechanical properties that compensated for the slender phenotype to establish normal strength.
Systematic Evaluation of Skeletal

Mechanical Function

\section{5}

Volume 3 


\section{Critical Parameters and Troubleshooting}

\section{General considerations}

The protocols in this manuscript are meant to provide a first-line phenotypic view of bone morphology and biomechnical characteristics to drive hypotheses and subsequent experiments for mechanistic investigations. To this end, the individual protocols were chosen to be accessible to researchers without extensive bioengineering backgrounds, although a certain level of familiarity in working with mouse bone, operating specialized machinery, and drawing appropriate conclusions for indepth investigations is required.

Ignoring technical expertise with specialized equipment, the fundamental steps for bone harvesting, mounting, and imaging are relatively straightforward, provided care is taken to follow the provided instructions. Please note, however, that these protocols do not discuss experimental design, and only touch on data interpretation. For additional insight into the many considerations required for detailed mouse bone experiments, refer to the references throughout this manuscript.

\section{Bone harvesting}

As mentioned above, bone harvesting is straightforward as long as one possesses the prerequisite knowledge of anatomy and exercises particular care to avoid shearing the epiphysis.

\section{Embedding in plastic}

When making MMA solutions, several safety guidelines bear repeating. All procedures must be performed under an approved chemical fume hood. Metal or other conductive instruments should never be used to weigh out benzoyl peroxide.

It is critical to avoid introducing water into any samples or MMA solutions, as this can interfere with the embedding process. Bottles containing MMA solutions should be thawed completely and warmed to room temperature before they are opened to avoid condensation. During the embedding process, extreme caution should be used to avoid introducing water into sample(s) after dehydration. All precautions described for preparing the MMA solutions (see Support Protocol) should be followed throughout the embedding procedure as well.

The embedding reaction should not be allowed to proceed too quickly, as this may cause the plastic to melt, generate bubbles, and damage the tissue. If at any point the embedding reaction proceeds too quickly, the MMA bottle (containing specimens) can be cooled at $-20^{\circ} \mathrm{C}$ to pause the polymerization process.

Keep in mind that the embedding protocol does not provide details for prior labeling of bones for epifluorescence microscopy. Refer to the protocol introduction for a brief overview of the labeling procedure we use, noting particularly that injection timing varies depending on the age of the mouse.

\section{Computed tomography}

For nanoCT imaging, as with all CT procedures, material selection and purity are critical. Most steps in this protocol require empirical testing to optimize settings to obtain the highest possible signal-to-noise ratio. The exact procedure outlined is tailored for the indicated scanning system and software, but the general concepts should be generally applicable.

\section{Bone bending tests}

Because bending tests are destructive, samples should be used for other (non-destructive) assays before beginning the bending tests. It is important to be thoroughly familiar with the selected testing machinery before beginning, follow strict sample organization/labeling procedures, and maintain sample hydration. Liberal use of practice dowels and other standards is strongly recommended to ensure that machinery parameters are appropriate and data recording is on-line.

\section{Ash weight, water content, and bone density}

Again, it is important to develop and validate a standard (such as an aluminum ring) that can be tested over time. Based on unpublished observations, the specific parameters for this protocol should work for both defatted and non-defatted mouse bone, but published results have (to date) been demonstrated only for the former. The protocol presented here is only appropriate if the overall specimen weight is greater than $8-10 \mathrm{mg}$.

\section{Anticipated Results}

The protocols within this manuscript are, as mentioned, meant to provide baseline insight into bone morphology and characteristics. It is, therefore, impractical to offer detailed anticipated results beyond examples provided by sample data (see Figs. 7, 8, 10, and 12). Ultimately, the order of procedures, detailed explanations, background information, and figures provide a framework for less-experienced investigators to establish fundamental skills and 
gather information to perform more mechanistic downstream studies.

\section{Time Considerations}

It is challenging to assign time frames for many of these protocols owing to the anticipated variance in experimenter experience, access to automated machinery, and familiarity/expertise with operating and maintaining advanced equipment. Assuming a moderate level of experience, bone harvesting is a relatively quick process, taking $\sim 5-10 \mathrm{~min}$ per mouse to harvest long bones and spine. Preparing the MMA solutions requires several days, although most of this comprises waiting time. Embedding bones is a timeconsuming process due to wait times, and several weeks should be allotted from beginning to end. Whole-bone mechanical testing is a relatively quick procedure, taking $\sim 5 \mathrm{~min}$ for a femur or spine. Tissue-level mechanical testing, however, is extremely time-consuming and requires tremendous attention to detail. This type of experiment requires $\sim 8 \mathrm{hr}$ of work for each bone sample. For ash content analysis, the individual weighing procedures are not time-consuming (1-2 min per sample), but the overall time needed is 3-5 days given the overnight drying and ashing times. Morphological analysis using the nanoCT is quite straightforward and requires $2-3 \mathrm{hr}$ to scan a bone, $10-15 \mathrm{~min}$ to reconstruct the $3 \mathrm{D}$ image, $1 \mathrm{hr}$ for calibration and image processing, and 10-20 min for analysis, depending on whether a femur or spine is being studied.

\section{Literature Cited}

Bonadio, J., Jepsen, K.J., Mansoura, M.K., Jaenisch, R., Kuhn, J.L., and Goldstein, S.A. 1993. A murine skeletal adaptation that significantly increases cortical bone mechanical properties. Implications for human skeletal fragility. J. Clin. Invest. 92:1697-1705.

Donovan, J. and Brown, P. 2006. Euthanasia. Curr. Protoc. Immunol. 73:1.8.1-1.8.4.

Furuya, K., Nifuji, A., Rosen, V., and Noda, M. 1999. Effects of GDF7/BMP12 on proliferation and alkaline phosphatase expression in rat osteoblastic osteosarcoma ROS 17/2.8 cells. $J$. Cell. Biochem. 72:177-180.
Jepsen, K.J. 2009. Systems analysis of bone. Wiley Interdiscip. Rev. Syst. Biol. Med. 1:7388.

Jepsen, K.J., Schaffler, M.B., Kuhn, J.L., Goulet, R.W., Bonadio, J., and Goldstein, S.A. 1997. Type I collagen mutation alters the strength and fatigue behavior of Mov13 cortical tissue. $J$. Biomech. 30:1141-1147.

Jepsen, K.J., Davy, D.T., and Krzypow, D.J. 1999. The role of the lamellar interface during torsional yielding of human cortical bone. $J$. Biomech. 32:303-310.

Jepsen, K.J., Hu, B., Tommasini, S.M., Courtland, H.-W., Price, C., Terranova, C.J., and Nadeau, J.H. 2007. Genetic randomization reveals functional relationships among morphologic and tissue-quality traits that contribute to bone strength and fragility. Mamm. Genome 18:492-507.

Jepsen, K.J., Hu, B., Tommasini, S.M., Courtland, H.-W., Price, C., Cordova, M., and Nadeau, J.H. 2009. Phenotypic integration of skeletal traits during growth buffers genetic variants affecting the slenderness of femora in inbred mouse strains. Mamm. Genome 20:21-33.

Jepsen, K.J., Courtland, H.-W., and Nadeau, J.H. 2010. Genetically-determined phenotype covariation networks control bone strength. $J$. Bone Miner. Res. 25:1581-1593.

Lou, J., Tu, Y., Burns, M., Silva, M.J., and Manske, P. 2001. BMP-12 gene transfer augmentation of lacerated tendon repair. J. Orthop. Res. 19:11991202.

Maloul, A., Rossmeier, K., Mikic, B., Pogue, V., and Battaglia, T. 2006. Geometric and material contributions to whole-bone structural behavior in GDF-7-deficient mice. Connect. Tissue Res. 47:157-162.

Marder, E. and Goaillard, J.M. 2006. Variability, compensation and homeostasis in neuron and network function. Nat. Rev. Neurosci. 7:563574.

Nadeau, J.H., Burrage, L.C., Restivo, J., Pao, Y.H., Churchill, G., and Hoit, B.D. 2003. Pleiotropy, homeostasis, and functional networks based on assays of cardiovascular traits in genetically randomized populations. Genome Res. 13:20822091.

Waddington, C.H. 1942. Canalization of development and the inheritance of acquired characters. Nature 14:563-565.

Wright, S. 1921. Correlation and causation. J. Agric. Res. 20:557-585.
Systematic

Evaluation of

Skeletal

Mechanical

Function

67

Volume 3 